\section{Response to Dissociation Between Severity of Takotsubo Cardiomyopathy and Presentation With Shock or Hypotension}

\author{
Chong CR et al. Clin Cardiol. 2013;36:401-406
}

\section{To the Editor:}

We read with great interest the article by Chong et $\mathrm{al}^{1}$ titled "Dissociation Between Severity of Takotsubo Cardiomyopathy and Presentation With Shock or Hypotension," which has recently been published in Clinical Cardiology.

The authors aimed to show the association between severity of early hypotension and the extent of myocardial involvement and dysfunction in patients with takotsubo cardiomyopathy (TTC). ${ }^{1}$ They used the Simpson biplane echocardiographic method for estimating left ventricular ejection fraction and analyzed wall motion scores according to a 17-segment model. They also quantified regional and global myocardial edema via T2-weighted cardiac magnetic resonance (CMR) imaging within 6 days of admission. In their study, the authors concluded that initial hypotension did not imply severe left ventricular inflammation or systolic dysfunction. Nonetheless, some comments may be beneficial.

The right ventricle (RV) may be predominantly involved in certain cardiac conditions such as arrhythmogenic right ventricular cardiomyopathy or TTC. In many congenital or acquired cardiac diseases primarily affecting the left ventricle, right heart involvement has an important role in prognosis of disease and is associated with a more severe impairment in left ventricular systolic function. The RV is difficult to evaluate using 2-dimensional (2D) imaging modalities due to its complex shape and orientation. ${ }^{2}$

Reversible RV dysfunction is common in TTC, and its presence can be associated with most severe left ventricular dysfunction and patients' clinical condition. CMR imaging has superiority in estimating and detecting RV involvement compared with $2 \mathrm{D}$ echocardiography. CMR imaging in the acute phase (at the time of initial clinical presentation) may provide more appropriate information about the structure and the functions of the RV. Nevertheless, due to the time delay between presentation and performing the CMR study in some patients (within 6 days), some wall motion abnormalities might be overlooked in the early period. ${ }^{3}$ Although T2-weighted imaging is the best approach for edema-weighted CMR imaging and has the capability of detecting increased myocardial water content, it has some disadvantages. T2-weighted imaging can detect the area of regional edema in the presence of an obvious normal myocardium as a reference segment. However, measuring the T2 signal may not be convenient in apical slices of patients with apical ballooning due to the absence of normal myocardial segment., ${ }^{4,5}$ Also, T1 mapping has superior diagnostic performance compared with the T2weighted methods in detecting myocardial edema. T1mapping imaging can be useful in detecting myocardial changes beyond those identified by wall motion abnormality and pathologies where edema may be diffuse and patchy. T1-weighted and T2-weighted mappings are complementary techniques to each other, and they must be used together to detect a cardiac abnormality.

Emre Yalcinkaya, MD

Murat Celik, MD

Uygar Yuksel, MD

Erkan Yildirim, MD

Department of Cardiology

Gulhane Military Medical Faculty

Ankara, Turkey

Baris Bugan, MD

Department of Cardiology

Malatya Army Hospital

Malatya, Turkey

\section{References}

1. Chong CR, Neil CJ, Nguyen TH, et al. Dissociation between severity of takotsubo cardiomyopathy and presentation with shock or hypotension. Clin Cardiol. 2013;36:401-406.

2. Tee M, Noble JA, Bluemke DA. Imaging techniques for cardiac strain and deformation: comparison of echocardiography, cardiac magnetic resonance and cardiac computed tomography. Expert Rev Cardiovasc Ther. 2013;11:221-231.

3. Haghi D, Fluechter S, Suselbeck T, et al. Cardiovascular magnetic resonance findings in typical versus atypical forms of the acute apical ballooning syndrome (Takotsubo cardiomyopathy). Int $J$ Cardiol. 2007;120:205-211.

4. Schmalfuss C. Tako-tsubo cardiomyopathy and cardiac magnetic resonance imaging. Clin Cardiol. 2011;34:145-146.

5. Ferreira VM, Piechnik SK, Dall'Armellina E, et al. Non-contrast T1-mapping detects acute myocardial edema with high diagnostic accuracy: a comparison to T2-weighted cardiovascular magnetic resonance. J Cardiovasc Magn Reson. 2012;14:42. 